\title{
Cardiac rehabilitation for older patients with cardiovascular pathology using robotic systems - A survey
}

\author{
Mihaela Mocan $^{1,2}$, Bogdan Mocan ${ }^{3}$
}

Corresponding author: Mihaela Mocan, E-mail: mihaela.mocan@gmail.com,

1. Internal Medicine Department, "Iuliu Hatieganu" University of Medicine and Pharmacy, Cluj-Napoca, Romania

2. Internal Medicine Department, Emergency Clinical County Hospital, Cluj-Napoca, Romania

3. Design Engineering and Robotics Department, Technical University of Cluj-Napoca, Romania

\begin{abstract}
In the last decades, we have witnessed a drastic increase in the proportion of elders in the western countries, and more than a third of worldwide population dies because of cardiovascular diseases. The elderly patients with cardiovascular diseases, but not only, are frail and need constant assistance, and so the healthcare system will slowly become incapable of properly address all the patients' medical problems and rehabilitation issues, while aiming towards a continuous increase of the life quality. The rehabilitation robotic systems have the potential to stimulate and support the re-learning of the functional motor pattern of over-ground walking in an optimal manner while increasing patient's motivation, which constitutes a crucial element in the subject's cardiac recovery. Thus, the present article aims at reviewing the robotic systems available for cardiac rehabilitation in elders with cardiac pathology.
\end{abstract}

Key words: cardiac rehabilitation, older patients, cardiovascular diseases, heart failure, rehabilitation robotic systems, assistive robotics,

\section{Introduction}

In the last decades, we have witnessed a drastic increase in the proportion of elders in the western countries, and more than a third of worldwide population dies because of cardiovascular diseases (1). Multiple recent investigations have documented an increase of $10 \%-25 \%$ in peak VO2 after exercise training in older patients with heart failure (2). The older patients with cardiovascular diseases, but not only, are frail and need constant assistance, and so the healthcare system will slowly become incapable of properly address all the patients' medical problems and rehabilitation issues, while aiming towards a continuous increase of the life quality $(3,4)$. An important part of cardiac rehabilitation programs includes cardiac training exercises recommended by a medical team and supervised by therapists. An important consideration when prescribing an exercise regimen in cardiac rehabilitation is the level of supervision required. Patients at moderate to high risk cardiac complication should participate in a medically supervised program with trained personnel and equipment suitable for advance cardiac life support. Moreover, this level of medical supervision should be continued for at least 8 to 12 weeks (5). In a society where there is a growing demand place on the healthcare system, the cardiac rehabilitation programs would represent a great financial burden. In UK, for example, the annual potential cost of cardiac rehabilitation was estimated of around $£ 42$ million (6) .In the era of telemedicine and robots, the supervision of exercise training could be undertaken by robotic systems (7).

In order to be able to address these needs, the European Commission has identified several major areas of interest where robotics would play an important role (8), among which:

- Rehabilitation robotics: refer to robotic system that would be used following a medical condition (traumatic, post-operative, cardiac or neurological) where their direct physical interaction with the patient would either enhance the recovery process or act as a replacement for a lost function.

- Assistive robotics: refer to secondary aspects related to the medical process, providing assistance to the healthcare givers of the patients.

The scientific literature has demonstrated that physical exercise training improves parameters like aerobic capacity and muscular strength in cardiac patients (9). Rehabilitation of cardiac patients has always been a great challenge, due to the complexity of the recovery treatment and so there has been a strong motivation to the development of new systems for increasing the efficiency of rehabilitation procedures (10). From this 
perspective, robotic assisted rehabilitation can be seen as an important tool that provide flexibility, customized training and enhanced recovery treatment for cardiac patients. However, as any other new technology, rehabilitation robotic systems should be refereed on its efficiency and costeffectiveness and not only on its technical performance. The rehabilitation robotic systems have the potential to stimulate and support the relearning of the functional motor pattern of overground walking in an optimal manner while increasing patient's motivation, which constitutes a crucial element in the subject's cardiac recovery (11).

Thus, the present article aims at reviewing the robotic systems available for cardiac rehabilitation in elders with cardiac pathology.

\section{A survey of rehabilitation robotic systems and their adequacy for the cardiac rehabilitation}

Repetitive task-oriented movements are the single most important variable of cardiac rehabilitation and have a therapeutic gain; the literature suggests that an elderly patient recovering from cardiac failure must play an active role in the rehabilitation process if improvement is to occur (9).

Several robotic systems designed for rehabilitation are presented in the scientific literature as prototypes while others are being on the market, already. Most of the developed robotic systems for rehabilitation were focus on neurological failure (ex. neuromuscular impairment) and less on heart failure. Furthermore, some of the robotic systems manufacturers defined heart failure as one of the exclusion criteria for robot-assisted gait therapy (Hocoma the Lokomat ${ }^{\circledR}$ system manufacturer) (10). In this paper we will analyse the rehabilitation robotic systems which allow an over-ground gait training without exoskeletal support. Assessing whether they can be used or were designed especially for patients with cardiac problems.

The Lokomat ${ }^{\circledR}$ robotic system is a bilaterally driven gait training equipment that is used in conjunction with a body-weight support system (12).

It combines the advantages of robotic orthoses with advanced feed-back algorithms and Virtual Reality applications while providing a safe environment for the patient. The advantage of Lokomat's training is to impress a correct physiological movement scheme, more repetition than any classical approach, ensuring a high patient comfort by placing the patient in a virtually pleasant environment. The robotic system supports the weight of the patient in a manner appropriate to the functional capacity of the subject, which leads to rapid and sustained progress. There is at least one research study that highlight Lokomat ${ }^{\circledR}$ robotic system can be used on cardiac rehabilitation procedures (10).

The SoloWalk ${ }^{\circledR}$ is one of the other rehabilitation robots that is aimed towards helping individuals suffering from neurological fails and movements disorders. SoloWalk ${ }^{\circledR}$ is powered by DC gearmotors for its omnidirectional wheels while a harness system, attached to a pair of underarm supports, is designed to lift the patients and prevent them from falling (13)

A force sensor is integrated between the main frame and the harness that measures interaction force generated by the patient's intention of movement. Patients are supposed to be able to walk freely with the assistance of the SoloWalk while the robot simply follows the patient's motion. Based on the SoloWalk characteristics and training possibilities we can say that the robotic system could be used for cardiac rehabilitation (13).

The KineAssist ${ }^{\circledR}$ consists of an omnidirectional base, harness joining at the pelvic, force torque sensor that measures interaction force generated by the patient's intention of movement, and an active body weight support (14). The robotic system provides stability of the patient, and sustaining body weight, allowing the pelvic movement without any restriction. Unique harness system protects patients from losing their balance by gripping them before they can fall, which increase confidence in their abilities while they growth endurance and strength.

The development of this robotic system has met the doctor's need as it generates an intense, specific locomotion pattern and walking training by supporting the patient body weight. To provide an effective rehabilitation training, the KineAssist ${ }^{\circledR}$ is capable of seven modes of operation that can be used throughout the rehabilitation therapy.

Robo- $\mathrm{K} \AA$, the walking rehabilitation robot developed by BA Healthcare is a prototype that helps patients to relearn to walk (15). This relearning process is basically limited by the patient's joint pains and the practitioners' available time. Due to the integrated intelligent suspension system that can relieve the patients from $50 \%$ of their weight, the 
stresses on their joints are significantly reduced.

Additionally, the robot localization system offers an autonomous navigation within rehabilitation centre, releasing the physiotherapist who can then focus on his therapeutic approach and on encouraging the patient to make progress. The rehabilitation robotic system, Robo- $K \AA$, is equipped with a recording system that store the parameters of the training devoted to each patient, offering them a progressive therapy, adapted accordingly to their own particularities and needs (15).

Some clinical trials were made at the University Hospital in Rennes on a sample of 36 patients using ROBO-K ${ }^{\circledR}$ and significant improvements have been seen regarding their walking performance (15).

The above presented four rehabilitation robotic systems illustrate very well the "over-ground gait training without exoskeletal support" device structure and the training possibilities for relearning to walk, or to increase the functional capacity of the subject, which leads to rapid and sustained progress. Theoretically, and in several cases also practically by medical trials it has been shown that these rehabilitation robotic systems can also be used for rehabilitating heart failure patients. Certainly, more studies need to be done.

\section{Discussions}

A variety of rehabilitation robotic systems were developed for and tested in clinical and rehabilitation environments. These systems have the potential to facilitate long time training, reproduce repetitive movements, motivate patients and reduce the therapists' work. New technologies made possible the improvement of the function, usability and accessibility of the robots in medical and social environment. Robots could provide accurate and standardized assessment methods for cardiac rehabilitation, as to compare intra and inter-subject performance. Furthermore, rehabilitations robotic systems could be the gate to advanced technologies which provide novel training programs for patients with cardiovascular diseases. Thus, virtual and augmented reality technologies associated to rehabilitation robotic systems could increase both the motivation to participate to therapy and the engagement during walking training. Sensor added to these robotic systems would give an accurate image of the biological parameters modified by constant training, to quantify the short and long-term benefits of cardiac rehabilitation programs.

The majority of rehabilitation robots were designed for intensive locomotor training in patients suffering a movement disorder after stroke or spinal cord injury. The rehabilitations robots useful for patients with stroke were elegantly reviewed and classified by Ghannadi B et al. (8).

The data regarding rehabilitation robots in cardiac pathology are scarce. The group who developed Lokomat robotic system designed a study in order to assess feasibility and safety of a robot-assisted gait therapy in patients early after open heart surgery (16). They showed that the "results with robotassisted training were comparable to early postoperative standard in hospital training and no deep sternal wound infection or any adverse event occurred in the robot- assisted training group" (16). Thus, the robot-assisted gait therapy with the Lokomat system proved to be feasible and safe for postoperative cardiac rehabilitation.

Another study, published recently, focused on the possibility to integrate a robotic agent into cardiac therapy (17). This work presents an exploratory experiment for on-line assessment of typical cardiac rehabilitation routines, and for integrating social robots in cardiac rehabilitation routine. Given the fact that cardiovascular patients, especially those diagnosed with heart failure have an increased psychological stress, rehabilitation robotic system could be helpful for the health care system, by precisely identifying risk factors, the potential risk of therapy and by increasing the patient's performance through motivation and engagement $(17,18)$.

\section{Future trends}

In the future, clinical and basic research is needed to investigate the role of rehabilitation robotic systems in cardiovascular patients. Close collaborations between clinical and basic research could improve the function of rehabilitations robotic system and the training protocols to obtain the best functional outcome for cardiac patients. Some parameters should be reconsidered such as: optimal training paradigms, duration, protocols parameters and the best way to integrate in both medical and social environment. Hopefully, the rehabilitation robotic systems might further help to investigate the cardiac rehabilitation benefits and the underlying mechanisms of recovery. 


\section{Declaration of conflict of interests}

The authors does not have any financial interest involving the companies and/or materials mentioned in this article.

\section{References}

1. Roth GA, Huffman MD, Moran AE, Feigin V, Mensah GA, Naghavi M, et al. Global and regional patterns in cardiovascular mortality from 1990 to 2013. Circulation. 2015; 132:1667-78.

doi:10.1161/CIRCULATIONAHA.114.008720.

2. Fleg JL. Exercise Therapy for Older Heart Failure Patients. Hear Fail Clin. 2017; 13:60717. doi:10.1097/NCN.0b013e3181a91b58.

3. Farcaş AD, Năstasă LE, Anton FP, Stoia MA, Goidescu M, Mocan Hognogi DL, et al. Quality of life - an important parameter of cardiac rehabilitation in heart failure patients. Balneo Res J 2018; 9:288-90.

doi: $\quad$ http://dx.doi.org/10.12680/balneo. 2018.198

4. Mocan M, Chiorescu R, Banc ON, Mocan B, Anton F, Stoia M, et al. Cardiac rehabilitation protocols outcome in frail patients undergoing transcatheter aortic valve implantation. Balneo Res J. 2018; 9:401-5. doi: http://dx.doi.org/10.12680/ balneo.2018.220.

5. Balady GJ, Ades PA, Bittner VA, Franklin BA, Gordon NF, Thomas RJ, et al. Referral, enrollment, and delivery of cardiac rehabilitation/secondary prevention programs at clinical centers and beyond: A presidential advisory from the American Heart Association. Circulation .2011; 124:2951-60. doi: 10.1161/CIR.0b013e31823b21e2.

6. Shields GE, Wells A, Doherty P, Heagerty A, Buck D, Davies LM. Cost-effectiveness of cardiac rehabilitation: a systematic review. Heart .2018; 104:1403-10. doi:10.1136/heartjnl-2017-312809.

7. Sitar-Taut AV, Sitar-Taut DA, Cramariuc O, Negrean V, Sampelean D, Rusu L, Orasan O, Fodor A, et al. Smart Homes for Older People Involved in Rehabilitation Activities - Reality or Dream, Acceptance or Rejection? Balneo Res J .2018; 9:291-8. doi: http://dx.doi.org/10.12680/ balneo.2018.199.
8. Ghannadi B, Sharif Razavian R, McPhee J. Upper extremity rehabilitation robots: a survey. Handb. Biomechatronics, Elsevier Inc. 2019, p. 319-53. doi:10.1016/B978-0-12-8125397.00012-X.

9. Da Gama AEF, Chaves $\mathrm{T}$ de $\mathrm{M}$, Fallavollita $\mathrm{P}$, Figueiredo LS, Teichrieb V. Rehabilitation motion recognition based on the international biomechanical standards. Expert Syst Appl .2019; 116:396-409. doi: 10.1016/j.eswa.2018.09.026.

10. Schoenrath F, Markendorf S, Brauchlin AE, Frank M, Wilhelm MJ, Saleh L, et al. Robotassisted training for heart failure patients - A small pilot study. Acta Cardiol .2015; 70:66571. doi:10.2143/AC.70.6.3120178.

11. Katahira R, Soga M. Development and Evaluation of a System for AR Enabling Realistic Display of Gripping Motions Using Leap Motion Controller. Procedia Comput Sci .2015; 60:1595-603. doi: 10.1016/j.procs.2015.08.269.

12. Lokomat 2019 [internet]. Available from: https://www.hocoma.com/solutions/lokomat/

13. SoloWalk 2019 [Internet]. Available from: https://www.designdirectory.com/federaldesign house/SoloWalk

14. HDT 2019 [Internet]. Available from: http://www.hdtglobal.com/product/kineassist/

15. THE MOBILITY BROUGHT TO HEALTHCARE 2019 [Internet]. Available from:http:/www.bahealthcare.eu/en/rehabilitatio n-robot-sofmer/

16. Schoenrath F, Markendorf S, Brauchlin AE, Seifert B, Wilhelm MJ, Czerny M, et al.

Robot-Assisted Training Early after Cardiac Surgery. J Card Surg. 2015; 30:574-80. doi:10.1111/jocs.12576.

17. Lara JS, Casas J, Aguirre A, Munera M, Rincon-Roncancio M, Irfan B, et al. Humanrobot sensor interface for cardiac rehabilitation. IEEE Int Conf Rehabil Robot. 2017: 1013-8. doi: 10.1109/ICORR.2017.8009382.

18. Fărcaş AD, Năstasă LE. Factors Influencing the Perception of Stress in Patients with Heart Failure. Procedia - Soc Behav Sci .2014; 127:144-8. doi: 10.1016/j.sbspro.2014.03.229. 